\title{
3 Research Soure

\section{WNT Signaling Influences Neurological Function and Psychiatric Disorders Through Regulating Glia Phenotypes and Neuron Plasticity}

\section{Haochen Wang}

Soochow University

Mengyang Yan

Soochow University Affiliated No 1 Peoplel's Hospital: First Affiliated Hospital of Soochow University

Zhiqi Cheng

Soochow University

Tongyu Rui

Soochow University

Yanan Yan

Soochow University

Jie Chen

Xi'an International Medical Center

\section{Zhiya Gu}

Soochow University

Li Hui

Soochow University Affiliated Guangji Hospital

Qiufang Jia

Soochow University Affiliated Guangji Hospital

\section{Xiping Chen}

Soochow University

Luyang Tao ( $\nabla$ taoluyang@suda.edu.cn )

Soochow University https://orcid.org/0000-0002-2440-2683

\section{Research article}

Keywords: astrocyte, WNT signaling pathway, co-culture, migration

Posted Date: June 7th, 2021

DOI: https://doi.org/10.21203/rs.3.rs-541915/v1 
License: (c) (i) This work is licensed under a Creative Commons Attribution 4.0 International License. Read Full License 


\section{Abstract}

Background: As an important signaling pathway during embryonic development, WNT/ $\beta$-catenin signaling regulates cell proliferation and differentiation. However, the role of WNT pathway on central nervous system (CNS) injury is not clear.

Methods: In this study, based on traumatic brain injury (TBI, in vivo) and acute neuroinflammation (in vivo and in vitro) models, WNT signaling regulating glia cell phenotypes and the fate of neurons was investigated, and the effect on neurological function and anxiety behavior were also studied. The intercellular interaction was verified by transwell experiments.

Results: The result showed that WNT signaling was inhibited at acute stage of brain injury and $\mathrm{TBI}$, and the expression of WNT/ $\beta$-catenin decreased in primary cultured neurons and astrocytes after lipopolysaccharide (LPS) treatment. WNT agonists ( $\mathrm{LiCl}$ and Wnt3a) treatment significantly relieved psychiatric symptoms post-TBI compared to vehicle-treated group. WNT agonist treatment accelerated the polarization of astrocytes and generated A2 astrocytes, and activation of WNT signaling in astrocytes could promote neuron axon formation and maintain neuronal stability.

Conclusions: Our findings revealed that WNT signaling could influence neuron plasticity through regulating the phenotypes of microglia and astrocytes, and thereby affect neurological function and anxiety state. This study provides an evidence that WNT signaling may be therapeutic target against psychiatric disorder after TBI

\section{Background}

Early views of glial cells are relatively inert cells, and now, glia are recognized as dynamic cells which respond to neuronal activity, sense and regulate metabolic changes[1]. Astrocytes, as the most abundant cells in the central nervous system (CNS), contribute to balance brain function and help to maintain the normal composition of the extracellular medium[2,3] and the perspective of microglia in disease development have evolved, which was seen to play crucial roles in promoting and limiting brain injury[4]. In traumatic brain injury (TBI) and other CNS diseases, the brain's innate response to injury is crucial, resident astrocytes and microglia are often the primary cell types to initiate an inflammatory cascade upon sensing danger, for this reason, the proteins which associated with the activation of these cells are often used as biomarkers[5].

CNS lesion will induce a generic response of reactive gliosis. Previous studies generally agreed that microglia have functional plasticity including M1 and M2 phenotypes. Recently, like microglia, A1 and A2 phenotype of astrocyte have been proved. A1 astrocytes increase many proinflammatory cytokines that shown negative function, however, A2 astrocytes upregulate neurotrophic factors for neuroprotective effect [6, 7]. Disturbances of normal neuron-astrocyte interactions lead to neurodegeneration and progression of neurological diseases [8]. 
WNTs are family of secreted lipid-modified signaling proteins which acting as short- or long-term signaling molecules in the regulation of cellular processes $[9,10]$. The WNT/ $\beta$-catenin signaling pathway is important for neurogenesis in the developing nervous system $[11,12]$. In present study, TBI (in vivo) and acute neuroinflammation (in vivo and in vitro) model were induced by $\mathrm{CCl}$ and lipopolysaccharide (LPS) respectively, the expression of WNT was detection in injured cortex. Meanwhile, WNT/B-catenin signaling regulating glia cell phenotypes and the fate of neurons was investigated, and the effect on neurological function and anxiety behavior were also studied.

\section{Materials And Methods}

\section{Animals}

The 8-week-old male Sprague-Dawley rats were used for animal experiments (SLAC Company, Shanghai, China). Animals were housed with a $12 \mathrm{~h}$ light/dark cycle and at $22{ }^{\circ} \mathrm{C}$ and allowed to free access food and water. All the animal procedures were approved by Institutional Animal Use and Care Committee at Soochow University and carrier out according to the guidelines of Animal Use and Care of the National Institutes of Health.

\section{Traumatic brain injury model}

TBI was performed using the controlled cortical impact (CCI) model as previously described[13].

\section{Acute neuroinflammation model}

Rats were anesthetized with $10 \%$ chloral hydrate $(0.35 \mathrm{ml} / 100 \mathrm{~g})$ and intracerebroventricular (ICV) injection of LPS was given using stereotaxic apparatus following conditions: $0.8 \mathrm{~mm}$ posterior to bregma; $1.5 \mathrm{~mm}$ lateral to saggital suture; $3.6 \mathrm{~mm}$ beneath the surface of brain [14].

\section{$\mathrm{LiCl}$ and Salinomycin treatment}

$\mathrm{LiCl}$ and salinomycin was dissolved in sterile saline, the final concentration was $8 \mathrm{mg} / \mathrm{ml}$ and $0.4 \mathrm{mg} / \mathrm{ml}$ respectively. $\mathrm{LiCl}$ or salinomycin was intraperitoneally injected $30 \mathrm{~min}$ post-TBI, followed by injections once daily for 3 days, 7 days or 14 days.

\section{Wnt3a and DKK-1 treatment}

Intranasal administration is a well-established non-invasive route for drug administration to the brain and allows for permeation of proteins and even cells across the blood-brain barrier (BBB)[15]. Recombinant Wnt3a and DKK-1 (R\&D Systems) was reconstituted at $10 \mathrm{ng} / \mu \mathrm{L}$ in phosphate-buffered saline (PBS) containing $0.1 \%$ BSA.

\section{Tissue collection}

Rats were sacrificed after 1 h, 6 h, 24 h, $3 d, 7 d, 14 d$ of TBI and LPS injection. Rats were anesthetized and scarified at different time-points, tissue samples from injured cerebral cortex were rapidly removed, 
and all the subgroup samples were snap frozen in liquid nitrogen and stored at $-80^{\circ} \mathrm{C}$ until be used.

\section{Primary astrocyte and microglia culture}

Primary astrocyte and microglia cultures were prepared from SD rats as described previously [16-18].

\section{Behavioral analysis}

Wire Grip Test (WGT) and Morris Water Maze (MWM) were chosen to explore ability of motor function, learning and memory after TBI. Open field test (OFT), light dark box test (LDB), marble burying test (MBT) was used to explore the anxiety-like behavior and sucrose preference test (SPT), forced swim test (FST) could reflect depression-like behavior. The detailed methods and protocols were shown in supplementary information.

\section{Primary neuron, astrocyte, microglia and co-culture}

Primary neuron cultures were prepared from SD rats and the detail was shown in supplementary information. The purification of astrocytes and microglia were plated in 6-wells dishes about the ratio of 3-5:1, the mature mixed glial cultures could be used after seven days. Primary astrocyte-neuron cocultures were prepared from SD rats as described previously[19].

\section{OGD and OGD/R establishment}

In oxygen/glucose deprivation injury and reperfusion (OGD/R) model, $2 \mathrm{mM} \mathrm{Na}_{2} \mathrm{~S}_{2} \mathrm{O}_{4}$ was employed to consumption oxygen [20]. The $\mathrm{Na}_{2} \mathrm{~S}_{2} \mathrm{O}_{4}$ was solution in glucose-free DMEM/F12 medium.

\section{Transwell analysis}

$0.4 \mu \mathrm{m}$ and $8 \mu \mathrm{m}$ pore diameter inserts were used in the experiment, the detailed process was shown in supplementary information.

\section{5-Ethynyl-2'-deoxyuridine (EdU) assay}

The proliferation of all type of astrocytes were measured by Cell-Light EdU Apollo567 In Vitro Kit (RiboBio, Guangzhou, China) following the manufacturer's instructions.

\section{Immunocytochemistry and Immunofluorescence staining}

The astrocytes were plated on the coverslips which have coated with poly-L-lysine. For Immunocytochemistry (ICC) [21], coverslips were prepared routinely, deparaffinized and rehydrated. Images were acquired using microscope (Nikco) and immunofluorescence staining was used to primary astrocyte-microglia co-culture, astrocytes scratch-wound model, different treatment groups of microglia and primary neuron culture in cells and rat brain tissue slices.

In this part, the primary antibodies were as follows: anti-GFAP (1:200, Abcam), anti-lba1 (1:100, Abcam), anti-MAP2 (1:100, Abcam).

\section{Western blot}


For cortical tissues, rats were sacrifice at $1 \mathrm{~h}, 6 \mathrm{~h}, 1 \mathrm{~d}, 3 \mathrm{~d}, 7 \mathrm{~d}$ and $14 \mathrm{~d}$ after TBI and LPS and cells samples were scraped from culture plate. The proteins of injured brain tissues and cells from different treatment groups were extracted by RIPA lysis. The proteins were separated by $8 \%, 10 \%$ and $12 \%$ SDSPAGE and transferred onto Hybond-Polyvinylidenedifluoride (PVDF) membranes.

The primary antibody involved in this paper were as follows: anti- $\beta$-catenin $(1: 1000, A b c a m)$, anti-II $\beta$ (1:500, Proteintech), anti-iNOS (1:500, CST), anti-AQP4 (1:500, Proteintech), anti- $\beta$-actin (1:5000, Abcam), anti-ß-tubulin (1:1000, Beyotime), anti-Bax (1:1000, Abcam), anti-Bcl2 (1:1000, Affinty).

\section{Real-time PCR}

Total RNA was extracted from primary cultured cells using Trizol reagent (Thermo, USA). The RNA sample were reverse-transcribed in $20 \mu \mathrm{l}$ at $42^{\circ} \mathrm{C}$ for $60 \mathrm{~min}$ then incubated at $70^{\circ} \mathrm{C}$ for $5 \mathrm{~min}$ and $4^{\circ} \mathrm{C}$ for $10 \mathrm{~min}$ according to the manufacturer's instructions (Thermo, USA). The sequences of the PCR primer are shown in supplementary information.

\section{Brain edema and Lesion volume assay}

Brain edema (water content) was measured as previously used. In brief, rats were anesthetized and sacrificed at $24 \mathrm{~h}$ and $3 \mathrm{~d}$ after TBI. The brain water content was measured with a drying method and percentage was calculated using the Elliott formula for each part:

$\%$ water $=\frac{\text { wet weight-dry weight }}{\text { wet } \text { weight }} \times 100 \%$.

Lesion volume was measured at $7 \mathrm{~d}$ post-TBI. Cryosections ( $15 \mu \mathrm{m}$ thick, $100 \mu \mathrm{m}$ intervals) were strained with H\&E photographed with light microscopy and an imaging program. Each section was measured for area with imageJ software.

\section{Statistics analysis}

All statistical analysis was performed using SPSS 24.0. The hapiro-Wilk test was used to evaluate whether the data were obedient to normal distribution. Difference between groups were calculate by $t$-test. Western blot was carried out with one-way or two-way ANOVA. For all comparisons, $P<0.05$ was regarded as statistically significant.

\section{Results}

\section{Activation of WNT signaling inhibit deficit of learning/memory and psychiatric disorder}

In behavior analysis, motor function of all the groups were recovery at 6 days after TBI and the rats treated with $\mathrm{LiCl}$ and Wnt3a could get high score at early-stage post TBI (Fig. 1B). In MWM test, TBI 
(vehicle) induced an increased latency at 11 to 21 days compared with the sham group, however, treatment with $\mathrm{LiCl}$ and Wnt3a reduced the latency on 13-21 days after TBI (Fig. 1C).

In OFT, the movement distance in TBI + salinomycin, TBI + DKK-1 group was significantly decrease at $3 \mathrm{~d}$ to $14 \mathrm{~d}\left(\mathrm{Fig} .1 \mathrm{D}_{1}-\mathrm{D}_{3}\right)$ post-TBI, however, the movement distance had no statistically significant at 28 days after TBI (Fig. 1D $\mathrm{D}_{4}$ ). Compare with sham group, the center distance of TBI, both in agonist and inhibitor group was downregulation at $3 d$ and $7 d$ after TBI (Fig. $1 E_{1-2}$ ), however, no statistically significant difference at $14 \mathrm{~d}$ to $28 \mathrm{~d}$ (Fig. $1 \mathrm{E}_{3-4}$ ). In LDB, as shown in Fig. $1 \mathrm{~F}$ and $1 \mathrm{G}$, light compartment times and number of light compartment entries significantly decreased if rats were suffered TBI. $\mathrm{LiCl}$ and Wnt3a could increase the light compartment time and entries compared with vehicle $\left(\mathrm{Fig} .1 \mathrm{~F}_{3}\right)$. The effects of $\mathrm{TBI}$, agonist and inhibitor treatments on MBT, which represent anxiety-related sequelae, are shown in Fig. $2 \mathrm{H}$. WNT agonist significantly decreased the marble burying behavior as compared to vehicle group at $7 \mathrm{~d}$ and $14 \mathrm{~d}$ post-TBI.

SPT could reflect depression-like behavior, significant decrease in sucrose consumption was observed in $\mathrm{TBI}, \mathrm{TBI}+\mathrm{DKK}$ and TBI + salinomycin groups at $7 \mathrm{~d}$ post-TBI $\left(\mathrm{Fig} .1 \mathrm{I}_{2}\right)$. Chronic treatment with Want3a and $\mathrm{LiCl}$ showed pronounced increase in sucrose consumption at $14 \mathrm{~d}$ post-TBI (Fig. $1 \mathrm{l}_{3}$ ). In the FST, TBI rats displayed despair behavior as increased time of immobility $\left(\right.$ Fig. $\left.1 \mathrm{~J}_{2-4}\right)$. TBI + Salinomycin and TBI + DKK increased time of immobility compared with sham groups, however, Wnt3a and LiCl decreased immobility time at $7 \mathrm{~d}$ to $14 \mathrm{~d}$ after TBI $\left(\mathrm{Fig} .1 I_{3-4}\right)$. In SIT, all the rats had no preference for 2 empty cages during a habituation period. When put a stranger rat in one cage, sham rats spend spent more time at ratcontaining cage until $14 \mathrm{~d}$ post-TBI compared with $\mathrm{TBI}+$ salinomycin and $\mathrm{TBI}+\mathrm{DKK}$ treatment (Fig. $1 \mathrm{~K}_{1}$ -

2). $\mathrm{LiCl}$ and Wnt3a could reverse the impaired social interaction at $7 \mathrm{~d}$ after TBI (Fig. $1 \mathrm{~K}_{2}$ ).

\section{Activation of WNT signaling benefit neuroprotection post- TBI}

We measured the level of $\beta$-catenin which is a key protein to regulate WNT signaling pathway. For injured cortex tissue, WNT signaling was dysregulated in TBI and acute neuroinflammation model (Supplementary Fig. 1A-B). In cell-cell interaction (Supplementary Fig. 1C), the WNT signaling was inhibited with activated microglia-condition medium (MCM) in primary neuron and slight change from 1 hour to 8 hours, but down-regulation at 12 hours in astrocyte (Supplementary Fig. 1D-E).

The neuroprotective effect was confirmed by lesion volume (LV) showed a significantly smaller LV in the $\mathrm{LiCl}$ and Wnt3a group on $14 \mathrm{~d}$ after TBI (Fig. 2A-B). In addition, brain edema was significantly decrease compared with TBI (vehicle) group at both $24 \mathrm{~h}$ and $3 \mathrm{~d}$ post TBI in agonists treatment groups (Fig. 2C). Then, in western blot (Fig. 2D-E), LiCl and Wnt3a, as agonists, could reversed the $\beta$-catenin decrease at 3 $d$ after TBI and the expression of inflammatory factors like iNOS and IL-1 $\beta$ was decrease in LiCl and Wnt3a treated groups compared with vehicle, salinomycin and DKK-1 group. The expression of Bcl-2 increased and Bax decreased at agonist groups. Immunofluorescence staining was employed to analyze the distribution and polarization state of astrocytes and microglia (Fig. 2F). Salinomycin and DKK 
decreased the number and length of branch in microglia and activated microglia. The level of microglia activation significantly increased compared with vehicle group (Fig. 2G). For astrocyte, the neurite length and cells' area also increased.

\section{Phenotype of astrocyte was regulated by microglia and WNT signaling pathway}

Under astrocytes-microglia/co-culture condition, the cells treated with LPS can induce the A1 astrocytes, in which the length of neurite increased and cells' area shrunk significantly (Fig. 3A-B). To analyze the relationship between microglia and WNT pathway, the primary microglia were treated with LPS/IL-4, $\mathrm{LiCl} / \mathrm{DKK}-1$, and OGD/R, our study found that activation of WNT signaling (LiCl treatment) in microglia will promote to generate M2-microglia (Fig. 3C) and activation of WNT signaling will promote CD206 upregulation and formation M2 microglia as well as reduce apoptosis (Fig. 3D-E).

We hypothesis different phenotype of microglia could modify reactivate astrocytes process. To prove the hypothesis, transwell was used to co-culture microglia and astrocytes. The microglia were plated in upper chamber and treated with LPS, II-4, DKK and LiCl, and then placed into the bottom wells that containing primary astrocytes (Fig. 3F). According to the western blot analysis, for astrocyte (Fig. 3G), $\beta$-catenin was overexpressed in $\mathrm{M} 2$ and $\mathrm{LiCl}$ treated microglia induced groups. Besides, iNOS, IL-1 $\beta$ and TNFa increased in M1- and DKK-microglia treated groups (especially DKK treated group), and AQP4 upregulated in the M1-microglia treated group significantly (Fig. 3H). Then, in RT-qPCR, C3 and GBP-2, regarded as A1 astrocyte markers, remarkably increased in M1- and DKK-microglia treatment group and $\mathrm{A} 2$ astrocyte markers S100a10, Ptx3, Tm4sf1, Arginase-1 and Nrf-2 were upregulated in M2- and LiCl-microglia treated groups compared with M1- and DKK-microglia treated groups (Fig. 3I).

\section{WNT/ $\beta$-catenin pathway regulated morphology and phenotype of astrocytes}

After treatment with LPS, we found morphological change in astrocytes (Fig. 4A). The area of astrocyte was significant decreased after treatment with LPS 12 hours and the neurite length increase, especially LPS induce 48 hours after LPS induction. To determine whether reactive astrocyte function changes or not, we examined levels of iNOS, AQP4, IL-1 $\beta$ and TNFa by western blot. The results revealed that iNOS and AQP4 increased at 12 hours post-treatment with LPS, while IL-1 $\beta$ increased at 8 hours, and TNFa increased at 1 hour (Fig. 4B-C).

The morphology of astrocytes was detected under different treatment condition (activated-MCM treatment $24 \mathrm{~h}, \mathrm{OGD}$, and OGD/R), astrocytes showed polygonal with the treatment of OGD, OGD/R and $\mathrm{LiCl}$ compared with treatment of activated MCM and DKK (Fig. 4D). Astrocytes suffered OGD, OGD/R and $\mathrm{LiCl}$ could activate the WNT/ $\beta$-catenin pathway and reduce the expression of AQP4 (Fig. 4E-F).

In recent research, double-immunofluorescence labeling with complement component 3 and glial fibrillary acidic protein (GFAP) had been used to label A1 astrocytes, and double-immunofluorescence with 
S100a10 and GFAP could labeling A2 astrocytes. Besides that, PTX3, TM4SF1, Nrf2 and Arginase-1 also overexpressed in A2 astrocytes, meanwhile, GBP2 could be used for A1 astrocytes marker. In RT-qPCR analysis, S100a10 and Tm4sf1 overexpressed in OGD/R and LiCl treatment groups and Arginase-1 and Ptx3 downregulated in LPS, activated-MCM and DKK-1 groups. Nrf-2 overexpressed in OGD/R and LiCl group, but there was no statistical significance in LiCl group. GBP2 and C3q increased significantly in activated-MCM and DKK-1 group (Fig. 4G).

WNT signaling could modify proliferation and migration of reactive astrocytes. To evaluate the relationship between phenotype of reactive astrocytes and WNT signaling pathway, the cell wound healing assays was used. Before scratch, all the groups were pre-treated with LPS, activated-MCM, LiCl, DKK-1 and OGD/R,the neurite length of the astrocytes was remarkable decreased in LiCl and OGD/R pretreated group (Supplementary Fig. 2A, 2D-E). In the meantime, the healed area is increased in activatedMCM and DKK treated group (Supplementary Fig. 2B and 2F). We hypothesized that the decrease in scratch area was due to inhibition of astrocyte proliferation. According to EdU assay we found the number of $\mathrm{EdU}^{+}$astrocytes were decrease in $\mathrm{LiCl}$ and OGD/R group (Supplementary Fig. 2C and 2G).

To assess the interaction among microglia, astrocyte and neuron, the transwell was employed to perform co-culture. Astrocytes and microglia were plated in upper chamber and neurons were plated in bottom chamber (Supplementary Fig. $2 \mathrm{H}$ ). The results showed that the migration of microglia had no change in all groups (Supplementary Fig. 2I-J) but the number of astrocytes migration was increased significantly in LPS treated neuron group. However, the astrocyte will lose the ability of migration if pre-treated with agonist $\mathrm{LiCl}$, no matter whether the neuron injured or not. The migration of astrocytes was weakened in OGD/R treated group compared with LPS and DKK group (Supplementary Fig. 2K).

\section{Different phenotype of astrocyte regulated neuron synapse formation and synapse elimination}

In primary neurons, there was no effect on length of synapsis in activation or inhibition WNT signaling, but activation WNT could promoted synapsis elimination and increased synapsis plasticity during neuron development (Fig. 5A-C). To investigate the effect of microglia and astrocytes on neuron synapse formation, the microglia were treated by LPS/IL-4 or LiCl/DKK firstly. All the types of microglia were plated in upper chamber, further, co-culture was performed after neuron plated in 6-wells culture dish for 36-48 h (Fig. 5D). $0.4 \mu \mathrm{m}$ and $8.0 \mu \mathrm{m}$ pore diameter inserts were performed for transwell assays. In 0.4 $\mu \mathrm{m}$ inserts, all the cells cannot migrate and $8.0 \mu \mathrm{m}$ can migrate randomly. We observed that the synapsis of primary neuron treated with M1- and DKK-microglia was inhibited and the number of synapsis increased compared with M2- and LiCl-microglia group, especially LiCl treatment group (Fig. 5E-F).

Then, all the type of astrocyte was plated in upper chamber, and co-culture was performed as above mentioned (Fig. 5G). We found that if reactive astrocytes lose the ability to migrate (astrocytes plated in $0.4 \mu \mathrm{m}$ inserts), the length of synapsis grew longer than reactive astrocytes plated in 8.0 chamber, especially in resting and $\mathrm{A} 2$ astrocytes (Fig. $5 \mathrm{H}$ ), meanwhile, the number of synapsis in neuron reduced significantly (Fig. 5I). If the primary neuron without astrocytes, the synapsis kept the level of complication 
and short (the data was not showed). In order to explore the regulation function between microgliaastrocyte-neuron axis and WNT pathway, primary astrocytes were plated in upper chamber and cocultured with bottom microglia. Then, the astrocytes were transferred to co-culture with primary neuron (Fig. 5J). If astrocytes induced by M2- and LiCl-microglia, the neuron showed longer synapsis, particularly in no migration condition (Fig. 5K-L).

It is known that limiting the migration of astrocytes can promote the neurons maturation. Apoptosis of neuron was aggravated by $\mathrm{A} 1$ astrocytes (activated-MCM) and DKK treatment, which showed the decrease of Bcl-2 vs Bax and increase of caspase-3. Similarly, astrocytes showed neuroprotective activity which induced by $\mathrm{M} 2$ and $\mathrm{LiCl}$-microglia, Bax and Caspase-3 were upregulated and $\mathrm{Bcl}-2$ was downregulated by M1- and DKK-microglia-astrocyte axis (Fig. 5M-P).

To further explore the effect of WNT-astrocyte-neuron axis, astrocyte-neuron direct co-culture was used to monitor neuron apoptosis. TUNEL positive cells were more abundant after LPS treatment. Meanwhile, $\mathrm{LiCl}$ could dramatically reduce the mix cells apoptosis. For OGD/R group, the number of astrocyte apoptosis was downregulated, but there has no difference (TUNEL positive cells) in primary neuron in all groups (Fig. 5Q-R).

\section{Discussion}

In our study, we elucidate a novel protective mechanism of WNT signaling activation against TBI induced deficit of neurological function and psychiatric disorder. We also provide evidence that activation of WNT signaling promotes recovery of anxiety- and depression-like behaviors, and relieves glia cells excessive polarization. Meanwhile, WNT signaling could modulate migration of astrocytes rather than microglia to affect plasticity of neuron. Based on primary cells culture, microglia with increased WNT could accelerate generation of A2 or resting-state astrocytes. Importantly, the results indicated that the astrocyte is a key role, which drive neuron stabilization, according to WNT signaling post CNS injuries.

WNT signaling affect a series of neurodevelopmental processes. Previous studies also revealed that the abnormal expression of WNT could lead to the disorder of cognitive function, deficit of learning and memory [12, 22] and may associate with mood disorder, such as schizophrenia [23], autism spectrum disorder [24] and bipolar disorder [25]. In our model, the behavioral tests were performed in this study, and the results showed that anxiety and depression significantly increased after TBI which also fits with recent reports implicating risk of anxiety and depression increased after TBI [26]. With treatment of DKK$1 /$ salinomycin, rats exhibited impaired anxiety, depression and social interaction behavior even lasted to 28 days.

The rodents exhibited cognitive dysfunction and activation of glia cells after TBI [27]. In our findings here, WNT agonists could inhibit activation of astrocytes and microglia, LiCl and Wnt3a inhibited microglia activation and decreased formation of reactive-astrocytes. Interestingly, the branch of microglia decreased and cell significantly swelled after WNT inhibitors treatment. To explain this phenomena, primary cells experiments were developed in this study. The results showed that the primary astrocyte 
reflected to WNT signaling later than neuron after MCM-LPS treatment. So, the mechanism of the glianeuron interaction induced by injury is emerged, a proposition, WNT signaling as key signaling regulate neuron through astrocyte and/or glia, is given, and the experiment are explored further.

Therefore, we firstly investigated the relationship between astrocytes-microglia interaction and WNT signaling. WNT-induced switch is an important in microglia M1/M2 phenotype regulation [28, 29]. In addition to the above findings, in this study, we found different phenotype of microglia would stimulate astrocytes polarization toward A1- and A2-astrocytes (resting-astrocyte) and activation of WNT affect phenotype of microglia, then, lead to change of astrocytes polarization further.

It has been revealed that reactive astrocytes are strongly induced by CNS injury and always occurs with brain tissue defect and A1 astrocytes was strongly associated with pathogenic progression and induced neuron deficit $[30,31]$. A1 astrocytes highly increase the complement component 3 (C3), histocompatibility 2, D region locus $1(\mathrm{H} 2 \mathrm{~d} 1)$ and serpin family G member 1 (Serping 1) [32]. A2 astrocytes upregulation many neurotrophic factors including pentraxin 3 (Ptx3), S100 calcium-binding protein A10 (S100a10) and sphingosine-1-phosphate receptor 3 (S1 pr3) [31, 32]. Recent study indicated that $\mathrm{A} 1$ reactive astrocytes could be induced only by activated microglia which induced by LPS [6], however, we achieved some new findings in this study. We detected the A1 and A2 specific markers including C3, GBP2, S100a10, Arginase-1, Ptx3, Tm4sf1 and Nrf-2. For A1 astrocyte markers, C3 and GBP2 increased in injury groups (treatment with LPS, LPS-MCM), meanwhile, inhibition of WNT signaling result in A1 astrocytes formation. A2 astrocyte markers (S100a10, Arginase-1, Ptx3, Tm4sf1 and Nrf-2) expressed high level in $\mathrm{OGD} / \mathrm{R}$ and $\mathrm{LiCl}$ treatment groups compared with injury groups. The finding means that activated WNT signaling is benefit to A2 astrocytes generation and WNT pathway could directly regulated polarization of astrocytes even without the exists of microglia. In vitro, astrocytes always present polygonal, bipolar and stellate. LPS could induced morphological changes in cultured astrocyte, and astrocytes' reactivity will be upregulated by the change and the percentage of cells with bipolar and stellate shapes were higher compared with resting astrocyte [33]. However, the morphological specificity of A1 and A2 astrocyte is still unknown. In our in vitro study, we found the morphology of A2 astrocytes were similar to resting-astrocyte, and activated WNT signaling pathway was benefit to maintain the condition of anti-inflammatory. A2 reactive astrocytes and resting astrocytes tended to format of polygonal fibroblast-like shape, however, the $\mathrm{A} 1$ reactive astrocytes are always bipolar and stellate. Compared with astrocytes in vitro, the cells from neonatal rodent brain show few synapse and present polygonal fibroblast-like shape under physiological condition and the change of astrocytes morphology such as the withdrawal or outgrowth of astrocyte neurites are expected to modify signal exchange between astrocyte and neuron. However, further study is needed.

Another interesting finding in our research is that $\mathrm{LiCl}$ and Wnt analogues have no significant effect on formation of synapses in primary neuron, but could promoted the synapsis elimination, and then, the M1and M2-like microglia could only regulate the formation of synapses. So, we assumed that the powerful ability of WNT signaling may relate to the function of secretion and metabolism of astrocytes, and the former hypothesis was proved by recent papers which have revealed that the release of glucose, 
glutamate and intermediary including cytokines and polypeptide were modified by WNT pathway to astrocyte [34,35]. It has become increasingly clear that not only based on the formation of synapses but also include the selective elimination of synaptic are essential for the development and maintenance of synaptic connectivity patterns [36-38]. So, we compared the effect of WNT-astrocyte/neuron and WNTmicroglia/astrocyte/neuron loop in synaptic plasticity, and found that WNT signaling could regulate phenotype of astrocytes and affected neuron function further. Activation of WNT signaling was benefit to maintain anti-inflammatory condition by transforming microglia from $M 1$ to $M 2$ phenotype, however, this indirect modulation led to weak influence for development and maturation of neurons. Early research revealed that astrocytes modulate neuron synapse function and development in cultured primary neurons, and the presence of astrocytes greatly enhance synaptic activity and promote response to neurotransmitters[37]. In this study, it is noteworthy that astrocytes, which WNT was activated, could contribute to neuron plasticity and synaptic elimination, meanwhile, activation of WNT in astrocytes decreased neuron apoptosis induced by LPS. Importantly, migration of astrocytes is disadvantage factor for neuron plasticity (Fig. 5H), but, WNT agonists can counteract the effect and contribute to neuron plasticity. For the reason, we believe that WNT-astrocyte/neuron loop is the most important mechanism in regulation of neuronal plasticity and function. Astrocyte-neuron crosstalk may be more essential mechanism in improving brain function and prevention of anxiety and other mental diseases after brain injury.

Moreover, many studies have proved that $\mathrm{A} 1$ astrocytes lose ability to induce synapse formation, and promote neuron death. However, A2 astrocytes obtain the capacity for function of phagocytosis and promote survivability of synaptic and neuronal $[6,39]$. In our experiments, the type A2 astrocytes which induced by WNT agonists, exert the greatest effects on the formation, development and recovery of damage neurons. It should be an important mechanism for protective effect in brain via regulation of astrocyte on WNT signaling. We also found that activation of WNT signaling pathway could inhibited the proliferation of primary astrocytes and maintained the integrity of damaged neuron (Fig. 6). This phenomenon may be related to the deficit of proliferation of astrocyte and inhibition of glial scar formation. It is a pity that $\mathrm{A} 2$ reactive astrocyte and resting state astrocyte have similar function in this study and it was difficult to distinguish A2- and resting state astrocyte on morphological level, protein and genes accurately and precisely. So, further studies are needed.

\section{Conclusions}

Overall, using multiple approaches, our research has identified that the activation of WNT pathway is implicated in neurofunctional recovery and activation of WNT pathway could relieve the psychiatric deficit post-TBI. Our findings support WNT/ $\beta$-catenin signaling pathway could affect the polarization of glial cells (astrocytes and microglia). Activation of WNT/ $\beta$-catenin signaling pathway could maintain the resting state of glial cells and promote the polarization of cells to anti-inflammatory. Glial cells play an important role in maintaining neuronal plasticity, and regulation mode of WNT/ $\beta$-catenin signaling pathway-astrocytes-neuron loop necessary for neuron plasticity. 


\section{Abbreviations}

TBI

Traumatic brain injury

\section{CNS}

Central nervous system

CCI

Controlled cortical impact

LPS

lipopolysaccharide

WGT

Wire Grip Test

\section{MWM}

Morris Water Maze

OFT

Open field test

MBT

Marble burying test

SPT

Sucrose preference test

FST

Forced swim test

OGD/R

Oxygen/glucose deprivation injury and reperfusion

Sal

Salinomycin

\section{Declarations}

\section{Ethics approval and consent to participate}

All studies involving animals were in accordance with $\mathrm{NIH}$ guidelines and all procedures were approved by Soochow University Animal Care and Use Committee.

\section{Consent for publication}

All authors have given their consent for publication.

\section{Availability of data and materials}

All data used and analyzed for the current study are available from the corresponding author on reasonable request. 


\section{Competing interests}

The authors have declared that no conflict of interest exists.

\section{Funding}

This study was supported by the National Nature Science Foundation of China (No. 81971800, 81871536, 81530062, 81871536, LYT and XPC) and PAPD (LYT).

\section{Author Contributions}

LYT, XPC and QFJ were responsible for the study concept and design. HCW, MYY and ZQC conducted the western blot and morphology experiments. HL, TYR and YNA performed behavioral analysis. ZYG, HCW performed primary cells culture. HCW and JC conducted transwell. All authors read and approved the final manuscript.

\section{Acknowledgements}

The authors thank all other members of the Tao lab for helpful discussion.

\section{Author details}

a Department of Forensic Medicine, School of basic medicine and Biological Sciences, Medical School of Soochow University, Suzhou, 215123, China. ${ }^{b}$ Department of Ophthalmology, The First Affiliated Hospital of Soochow University, Shizi Street 188, Suzhou, 215006, China. ${ }^{c}$ XI'an international medical center hospital, XI'an, 710100, China. ${ }^{d}$ Affiliated Guangji Hospital, Soochow University, Suzhou, 215137, China

\section{References}

1. Barros LF, Brown A, Swanson RA. Glia in brain energy metabolism: A perspective. Glia. 2018;66:1134-7.

2. Zeug A, Muller FE, Anders S, Herde MK, Minge D, Ponimaskin E, Henneberger C. Control of astrocyte morphology by Rho GTPases. Brain Res Bull. 2018;136:44-53.

3. Chen J, Zhang DM, Feng X, Wang J, Qin YY, Zhang T, Huang Q, Sheng R, Chen Z, Li M, Qin ZH. TIGAR inhibits ischemia/reperfusion-induced inflammatory response of astrocytes. Neuropharmacology. 2018;131:377-88.

4. Li Q, Barres BA. Microglia and macrophages in brain homeostasis and disease. Nat Rev Immunol. 2018;18:225-42.

5. Karve IP, Taylor JM, Crack PJ. The contribution of astrocytes and microglia to traumatic brain injury. Br J Pharmacol. 2016;173:692-702.

6. Liddelow SA, Guttenplan KA, Clarke LE, Bennett FC, Bohlen CJ, Schirmer L, Bennett ML, Munch AE, Chung WS, Peterson TC, et al. Neurotoxic reactive astrocytes are induced by activated microglia. 
Nature. 2017;541:481-7.

7. Stogsdill JA, Ramirez J, Liu D, Kim YH, Baldwin KT, Enustun E, Ejikeme T, Ji RR, Eroglu C. Astrocytic neuroligins control astrocyte morphogenesis and synaptogenesis. Nature. 2017;551:192-7.

8. Wakida NM, Cruz GMS, Ro CC, Moncada EG, Khatibzadeh N, Flanagan LA, Berns MW. Phagocytic response of astrocytes to damaged neighboring cells. PLoS One. 2018;13:e0196153.

9. Zhang YK, Huang ZJ, Liu S, Liu YP, Song AA, Song XJ. WNT signaling underlies the pathogenesis of neuropathic pain in rodents. J Clin Invest. 2013;123:2268-86.

10. Yang J, Zhang X, Wu Y, Zhao B, Liu X, Pan Y, Liu Y, Ding Y, Qiu M, Wang YZ, Zhao G. Wnt/beta-catenin signaling mediates the seizure-facilitating effect of postischemic reactive astrocytes after pentylenetetrazole-kindling. Glia. 2016;64:1083-91.

11. Zhang L, Yan R, Zhang Q, Wang H, Kang X, Li J, Yang S, Zhang J, Liu Z, Yang X. Survivin, a key component of the Wnt/beta-catenin signaling pathway, contributes to traumatic brain injury-induced adult neurogenesis in the mouse dentate gyrus. Int J Mol Med. 2013;32:867-75.

12. Chen J, Wang H, Luo C, Gao C, Zhang Y, Chen G, Chen W, Chen X, Tao L. Chd8 Rescued TBI-Induced Neurological Deficits by Suppressing Apoptosis and Autophagy Via Wnt Signaling Pathway. Cell Mol Neurobiol. 2020;40:1165-84.

13. Gao C, Wang H, Wang T, Luo C, Wang Z, Zhang M, Chen X, Tao L. Platelet regulates neuroinflammation and restores blood-brain barrier integrity in a mouse model of traumatic brain injury. J Neurochem. 2020;154:190-204.

14. Sharma M, Gupta YK. Intracerebroventricular injection of streptozotocin in rats produces both oxidative stress in the brain and cognitive impairment. Life Sci. 2001;68:1021-9.

15. Zhang JY, Lee JH, Gu X, Wei ZZ, Harris MJ, Yu SP, Wei L. Intranasally Delivered Wnt3a Improves Functional Recovery after Traumatic Brain Injury by Modulating Autophagic, Apoptotic, and Regenerative Pathways in the Mouse Brain. J Neurotrauma. 2018;35:802-13.

16. Kumamaru H, Saiwai H, Kobayakawa K, Kubota K, van Rooijen N, Inoue K, Iwamoto Y, Okada S. Liposomal clodronate selectively eliminates microglia from primary astrocyte cultures. J Neuroinflammation. 2012;9:116.

17. Lin L, Desai R, Wang X, Lo EH, Xing C. Characteristics of primary rat microglia isolated from mixed cultures using two different methods. J Neuroinflammation. 2017;14:101.

18. Tamashiro TT, Dalgard CL, Byrnes KR. Primary microglia isolation from mixed glial cell cultures of neonatal rat brain tissue. J Vis Exp 2012:e3814.

19. Oyanagi K, Tashiro T, Negishi T. Cell-type-specific and differentiation-status-dependent variations in cytotoxicity of tributyltin in cultured rat cerebral neurons and astrocytes. J Toxicol Sci. 2015;40:45968.

20. Wang XQ, Yao RQ, Liu X, Huang JJ, Qi DS, Yang LH. Quercetin protects oligodendrocyte precursor cells from oxygen/glucose deprivation injury in vitro via the activation of the PI3K/Akt signaling pathway. Brain Res Bull. 2011;86:277-84. 
21. Senthilkumaran C, Hewson J, Ollivett TL, Bienzle D, Lillie BN, Clark M, Caswell JL. Localization of annexins $A 1$ and $A 2$ in the respiratory tract of healthy calves and those experimentally infected with Mannheimia haemolytica. Vet Res. 2015;46:6.

22. Yang Y, Cheng Z, Tang H, Jiao H, Sun X, Cui Q, Luo F, Pan H, Ma C, Li B. Neonatal Maternal Separation Impairs Prefrontal Cortical Myelination and Cognitive Functions in Rats Through Activation of Wnt Signaling. Cereb Cortex. 2017;27:2871-84.

23. Bem J, Brozko N, Chakraborty C, Lipiec MA, Kozinski K, Nagalski A, Szewczyk LM, Wisniewska MB. Wnt/beta-catenin signaling in brain development and mental disorders: keeping TCF7L2 in mind. FEBS Lett. 2019;593:1654-74.

24. Vallee A, Vallee JN, Lecarpentier Y. PPARgamma agonists: potential treatment for autism spectrum disorder by inhibiting the canonical WNT/beta-catenin pathway. Mol Psychiatry. 2019;24:643-52.

25. Mulligan KA, Cheyette BN. Neurodevelopmental Perspectives on Wnt Signaling in Psychiatry. Mol Neuropsychiatry. 2017;2:219-46.

26. Figueiredo TH, Harbert CL, Pidoplichko V, Almeida-Suhett CP, Pan H, Rossetti K, Braga MFM, Marini AM. Alpha-Linolenic Acid Treatment Reduces the Contusion and Prevents the Development of Anxiety-Like Behavior Induced by a Mild Traumatic Brain Injury in Rats. Mol Neurobiol. 2018;55:187200.

27. Liu ZM, Chen QX, Chen ZB, Tian DF, Li MC, Wang JM, Wang L, Liu BH, Zhang SQ, Li F, et al. RIP3 deficiency protects against traumatic brain injury (TBI) through suppressing oxidative stress, inflammation and apoptosis: Dependent on AMPK pathway. Biochem Biophys Res Commun. 2018;499:112-9.

28. L'Episcopo F, Tirolo C, Serapide MF, Caniglia S, Testa N, Leggio L, Vivarelli S, Iraci N, Pluchino S, Marchetti B. Microglia Polarization, Gene-Environment Interactions and Wnt/beta-Catenin Signaling: Emerging Roles of Glia-Neuron and Glia-Stem/Neuroprogenitor Crosstalk for Dopaminergic Neurorestoration in Aged Parkinsonian Brain. Front Aging Neurosci. 2018;10:12.

29. Zhang D, Lu Z, Man J, Cui K, Fu X, Yu L, Gao Y, Liao L, Xiao Q, Guo R, et al. Wnt-3a alleviates neuroinflammation after ischemic stroke by modulating the responses of microglia/macrophages and astrocytes. Int Immunopharmacol. 2019;75:105760.

30. Qian D, Li L, Rong Y, Liu W, Wang Q, Zhou Z, Gu C, Huang Y, Zhao X, Chen J, et al. Blocking Notch signal pathway suppresses the activation of neurotoxic $A 1$ astrocytes after spinal cord injury. Cell Cycle. 2019;18:3010-29.

31. Xu X, Zhang A, Zhu Y, He W, Di W, Fang Y, Shi X. MFG-E8 reverses microglial-induced neurotoxic astrocyte (A1) via NF-kappaB and PI3K-Akt pathways. J Cell Physiol. 2018;234:904-14.

32. Su Y, Chen Z, Du H, Liu R, Wang W, Li H, Ning B. Silencing miR-21 induces polarization of astrocytes to the $A 2$ phenotype and improves the formation of synapses by targeting glypican 6 via the signal transducer and activator of transcription-3 pathway after acute ischemic spinal cord injury. FASEB J. 2019;33:10859-71. 
33. Acaz-Fonseca E, Ortiz-Rodriguez A, Azcoitia I, Garcia-Segura LM, Arevalo MA. Notch signaling in astrocytes mediates their morphological response to an inflammatory challenge. Cell Death Discov. 2019;5:85.

34. Brekke E, Berger HR, Wideroe M, Sonnewald U, Morken TS. Glucose and Intermediary Metabolism and Astrocyte-Neuron Interactions Following Neonatal Hypoxia-Ischemia in Rat. Neurochem Res. 2017;42:115-32.

35. Cisternas P, Zolezzi JM, Martinez M, Torres VI, Wong GW, Inestrosa NC. Wnt-induced activation of glucose metabolism mediates the in vivo neuroprotective roles of Wnt signaling in Alzheimer disease. J Neurochem. 2019;149:54-72.

36. Paixao S, Klein R. Neuron-astrocyte communication and synaptic plasticity. Curr Opin Neurobiol. 2010;20:466-73.

37. Chung WS, Allen NJ, Eroglu C. Astrocytes Control Synapse Formation, Function, and Elimination. Cold Spring Harb Perspect Biol. 2015;7:a020370.

38. Blanco-Suarez E, Caldwell AL, Allen NJ. Role of astrocyte-synapse interactions in CNS disorders. J Physiol. 2017;595:1903-16.

39. Clarke LE, Liddelow SA, Chakraborty C, Munch AE, Heiman M, Barres BA. Normal aging induces A1like astrocyte reactivity. Proc Natl Acad Sci U S A. 2018;115:E1896-905.

\section{Figures}




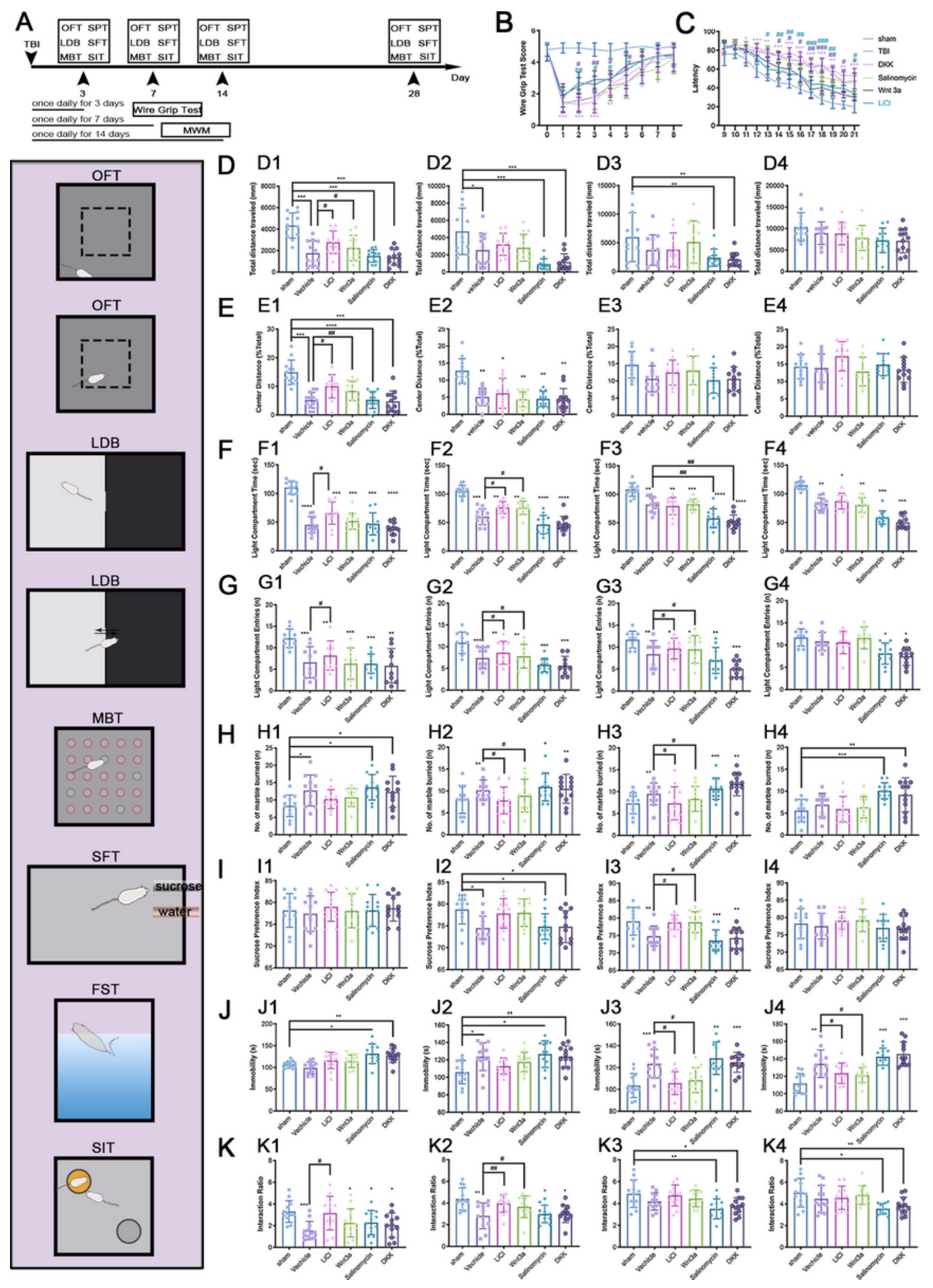

Figure 1

Traumatic brain injury induce anxiety- and depression-like behaviors in rats. (A) Time line of the experiments. (B) Wire grip test was used to determine the change of motor function. $\left({ }^{*} p<0.05,{ }^{* *} p<0.01\right.$, *** $p<0.001$ vs sham rats, \#p<0.05, \#\# $p<0.01$, \#\#\# $p<0.01$ vs vehicle, $n=6)$. (C) The MWM was performed to analyze the change of spatial memory in groups of sham, vehicle, DKK, LiCl, Salinomycin, and Wnt3a $(n=15)$. (D-E) Anxiety-like behavior was determined through open field test (total distance traveled) at $3 \mathrm{~d}$, 
$7 \mathrm{~d}, 14 \mathrm{~d}$ and $28 \mathrm{~d}$ after TBI. The percentage of center distance in open field test was analyzed at $7 \mathrm{~d}, 14 \mathrm{~d}$ and $21 \mathrm{~d}$ after TBI. ( ${ }^{*} p<0.05$, ** $p<0.01,{ }^{\star \star *} p<0.001$ vs sham rats, $\# p<0.05, \# \# p<0.01$ vs vehicle, $n=12$ ). (F-G) Anxiety-like behavior was determined through light dark box test was explored at $3 \mathrm{~d}, 7 \mathrm{~d}, 14 \mathrm{~d}$ and $28 \mathrm{~d}$ after TBI. The light compartment times and entries was recorded. ( ${ }^{*} p<0.05$, ${ }^{* \star} p<0.01,{ }^{* \star *} p<0.001$ vs sham rats, $\# p<0.05, \# \# p<0.01$ vs vehicle, $n=12)$. (H) Marble Burying Test was used to analyze anxiety post-TBI. $\left({ }^{*} p<0.05,{ }^{*} p<0.01, * \star \star p<0.001\right.$ vs sham rats, $\# p<0.05, \# \# p<0.01, \# \# \# p<0.01$ vs vehicle, $n=12)$. (I) The sucrose preference test was performed for detected depression after TBI. $\left({ }^{\star} p<0.05\right.$, * $p<0.01, * \star \star p<0.001$ vs sham rats, $\# p<0.05, \# \# p<0.01$, \#\#\# p<0.01 vs vehicle, $n=12)$. (J) Forced Swim Test could reflect despair with rats suffered TBI. $\left({ }^{*} p<0.05,{ }^{* *} p<0.01, * \star \star p<0.001\right.$ vs sham rats, $\# p<0.05$, \#\# $p<0.01$, \#\#\# $<<0.01$ vs vehicle, $n=12$ ). (K) In Social Interaction Test, the interaction ratio with stranger rats were determined. $\left({ }^{\star} p<0.05,{ }^{\star *} p<0.01,{ }^{\star \star \star} p<0.001\right.$ vs sham rats, $\# p<0.05, \# \# p<0.01, \# \# \# p<0.01$ vs vehicle, $n=12$ ) 


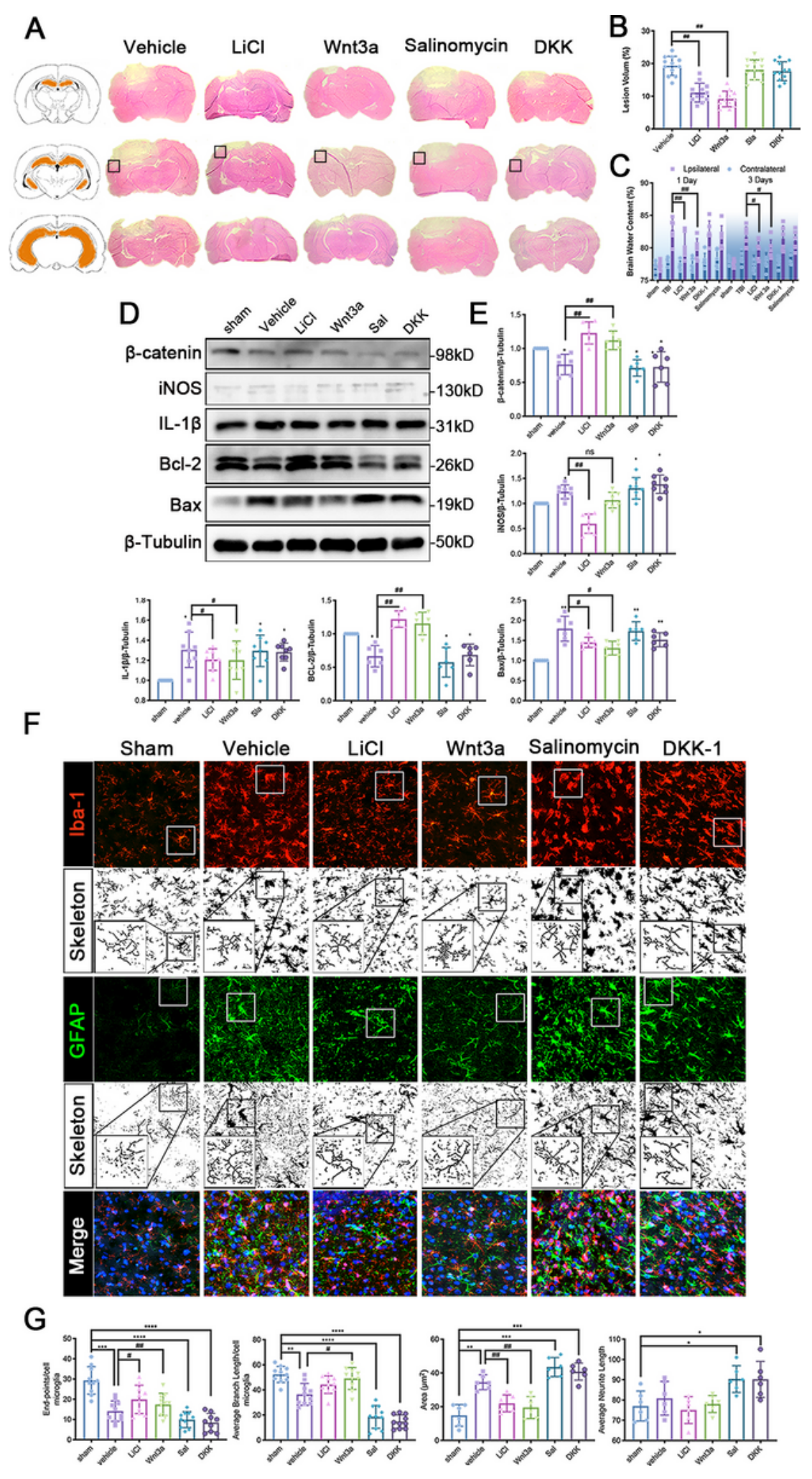

\section{Figure 2}

Activation of WNT signaling suppressed apoptosis and improve nerve function. (A) Representative images of H\&E staining brain slices on $14 \mathrm{~d}$ post-TBI. (B) The quantitative analysis of brain lesion volume (\#\# $p<0.01$, vs vehicle group). (C) The percentage of brain water content correlation with WNT signaling in ipsilateral hemisphere after TBI $1 d$ and $3 d(\# p<0.05$, \#\# $p<0.01$, vs vehicle group, $n=6)$. (D) The expression level of $\beta$-catenin, iNOS, IL-1 $\beta$, Bcl-2 and Bax in brain cortical tissue post-TBI ( $3 \mathrm{~d}$ ) was 
analyzed. (E) For western blot, ${ }^{\star} p<0.05$, ${ }^{\star \star} p<0.01$, vs sham group (control group); $\# p<0.05$, \#\# $p<0.01$, vs vehicle group, $n=6$. $(F)$ immunofluorescence staining was employed to determine the number and morphology of astrocytes and microglia. (G) End-point and branch length of microglia as well as area and neurite length of astrocyte was analyzed. $\left({ }^{\star} p<0.05,{ }^{\star *} p<0.01\right.$, vs sham group (control group); $\# p<0.05$, \#\# $p<0.01$, vs vehicle group, $n=6$ ).

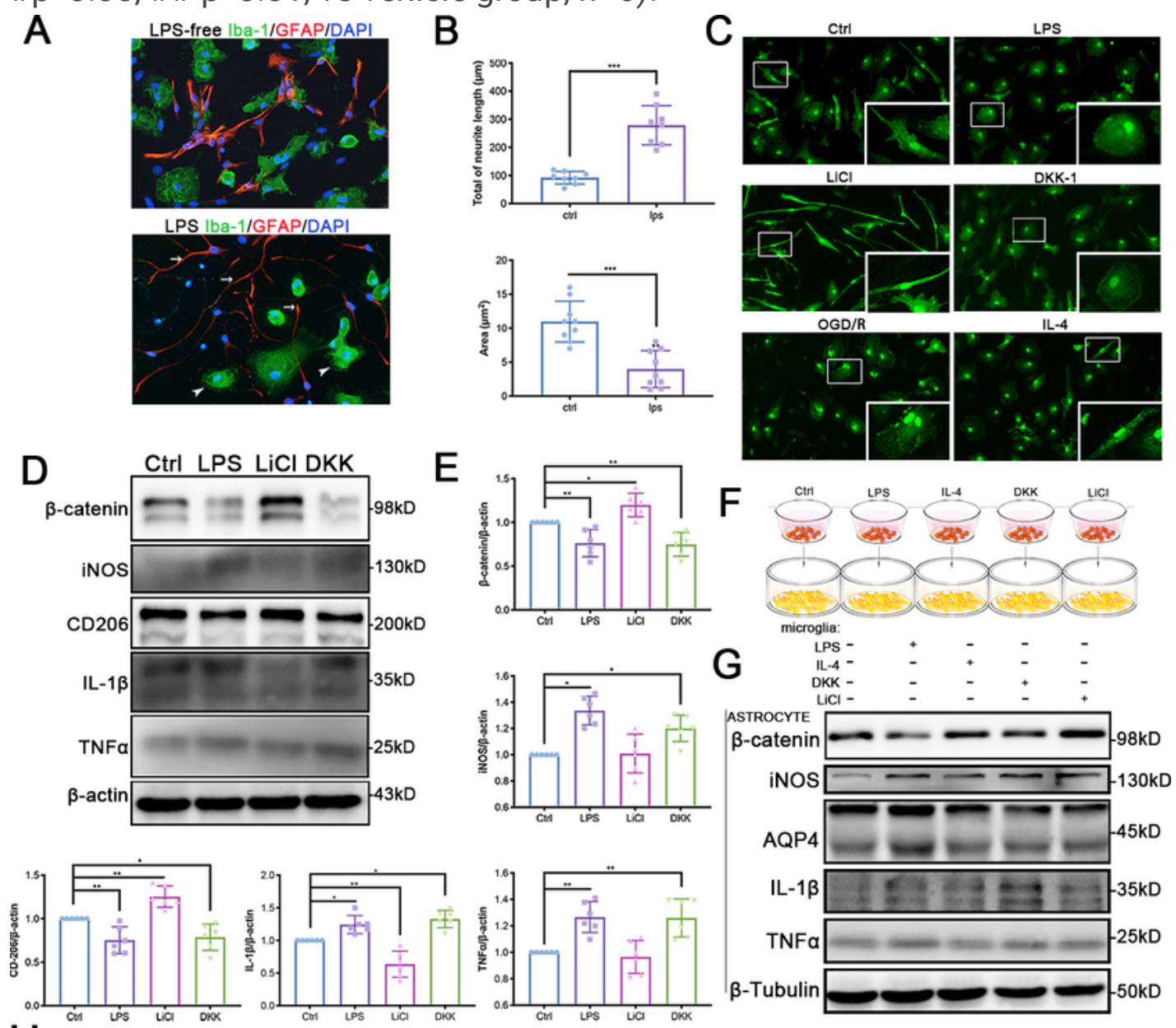

$\mathrm{H}$
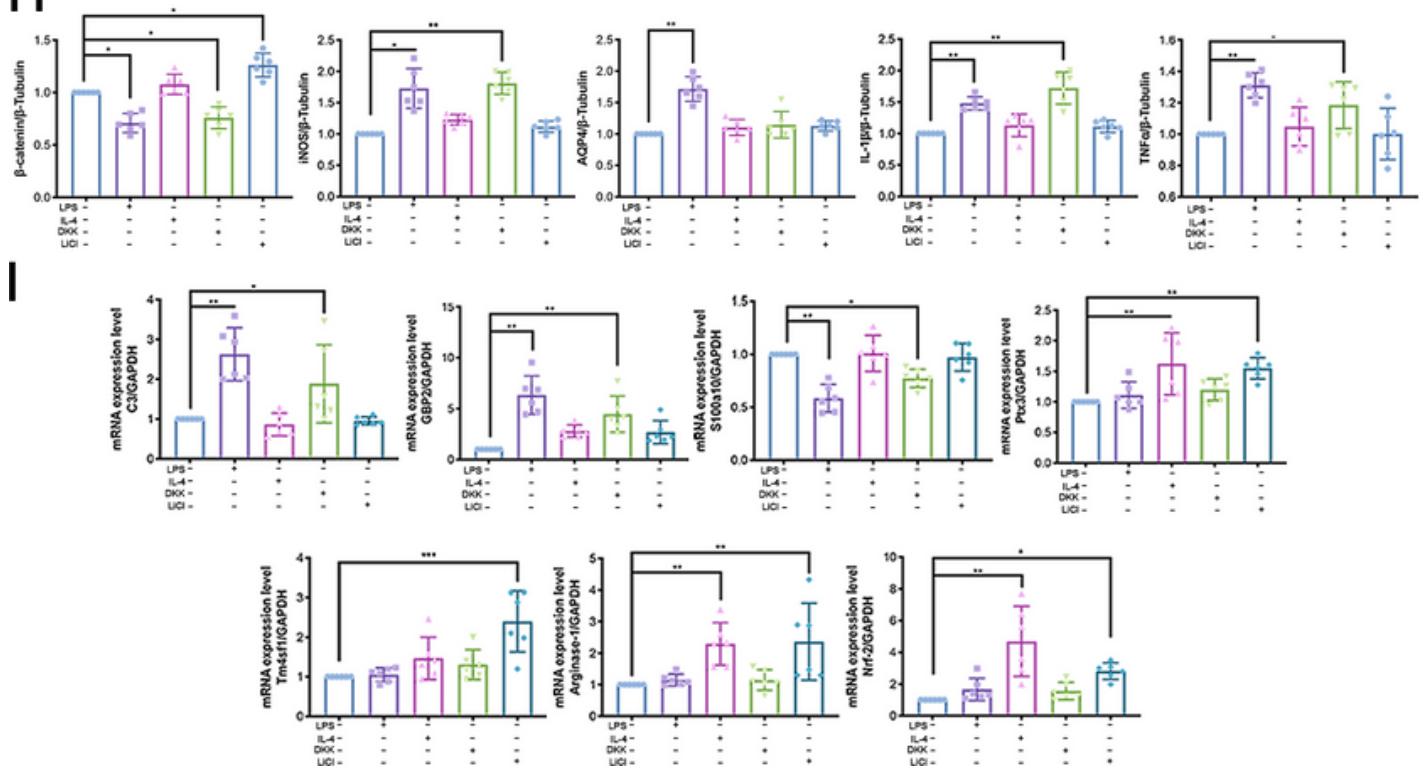

Figure 3 
LPS and WNT signaling regulated morphology of astrocytes and state of activation. (A) Morphological change of astrocytes exposed to LPS at different time points. ( $n=6$ independent cells preparations). (B) The expression level of iNOS, AQP4, IL-1 $\beta$ and TNFa in cultured astrocytes at different time points after LPS treatment. (C) ${ }^{*} p<0.05, * * p<0.01$ vs no treated groups ( $n=3-6$ independent cells preparations). (D) Correlation between the morphological change of astrocytes and WNT pathway, $n=6$ independent cells preparations. (E) The expression of $\beta$-catenin, iNOS, AQP4, IL-1 $\beta$, and TNFa with different type of astrocytes. $(F){ }^{*} p<0.05, * \star p<0.01$ vs activated-MCM groups, $n=3-6$ independent cells preparations. $(G)$ The mRNA expression level S100a10, Ptx3, Tm4sf1, Aginase-1, Nrf-2, C3q and GBP2 under different treatment condition.. ${ }^{*} p<0.05,{ }^{\star *} p<0.01,{ }^{\star \star \star} p<0.001$ vs control group, $n=3-6$ independent cells preparations. 

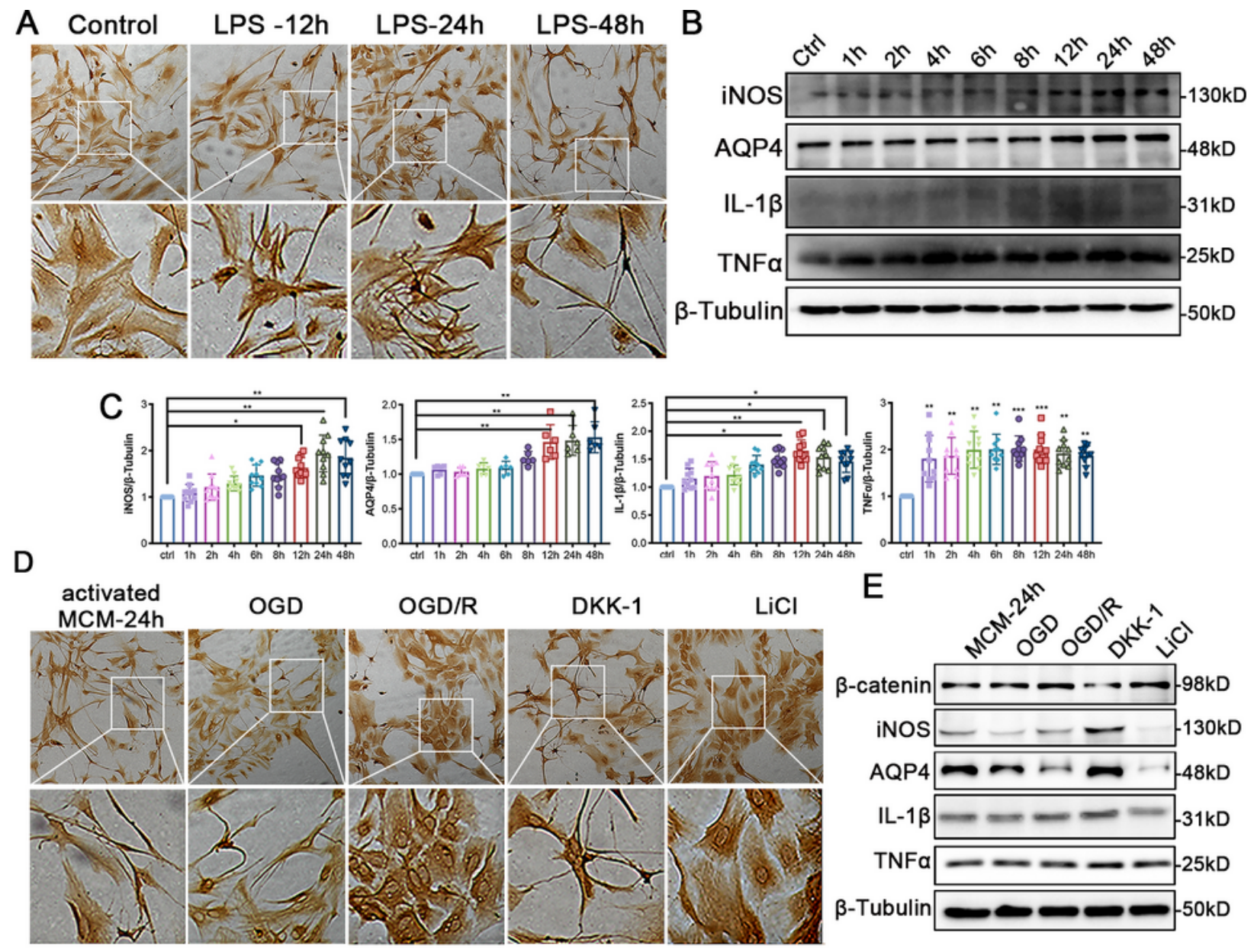

$\mathrm{F}$
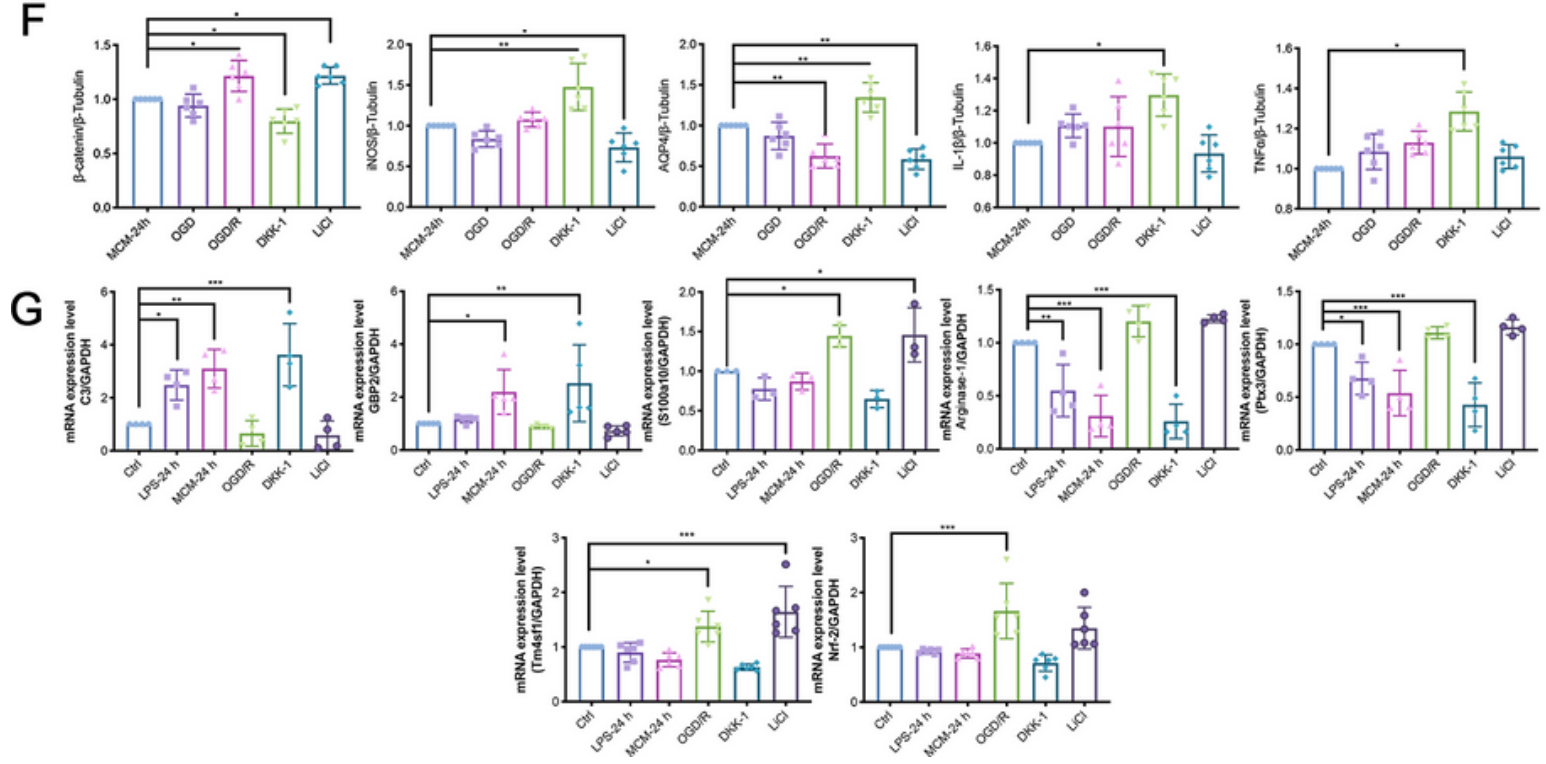

Figure 4

WNT signaling regulates microglia polarization and then affects the activation of astrocytes. (A) Astrocytes and microglia co-culture with or without LPS treatment, $\mathrm{n}=3$ independent cells preparations. (B) Measurement of neurite length and area of astrocytes in co-culture system, $\star \star \star ~ p<0.01$ vs control group (LPS-free), n=3-6 independent cells preparations. (C) Microglia treated with LPS, LiCl, DKK-1, IL-4 and OGD/R ( $n=3$ independent cells preparations). (D) The $\beta$-catenin, iNOS, CD206, IL-1 $\beta$ and TNFa 
expressed in microglia with LPS, LiCl and DKK treatment. (E) ${ }^{*} p<0.05,{ }^{*} p<0.01$ vs none treated control group ( $n=3$ independent cells preparations). ( $F$ ) The experimental design to detect the effect of different type of microglia on astrocyte. (G) The expression of $\beta$-catenin, iNOS, AQP4, IL-1 $\beta$, and TNFa were induced by different type of microglia. $(H) * p<0.05, * * p<0.01$ vs none treated control group $(n=3$ independent cells preparations). (I) The mRNA expression level of S100a10, Ptx3, Tm4sf1, Aginase-1, Nrf$2, \mathrm{C} 3 \mathrm{q}$ and GBP2 under different condition of treatment. ${ }^{\star} p<0.05$, ${ }^{\star \star} p<0.01$, ${ }^{\star \star \star} p<0.001$ vs control group ( $n=3-6$ independent cells preparations).
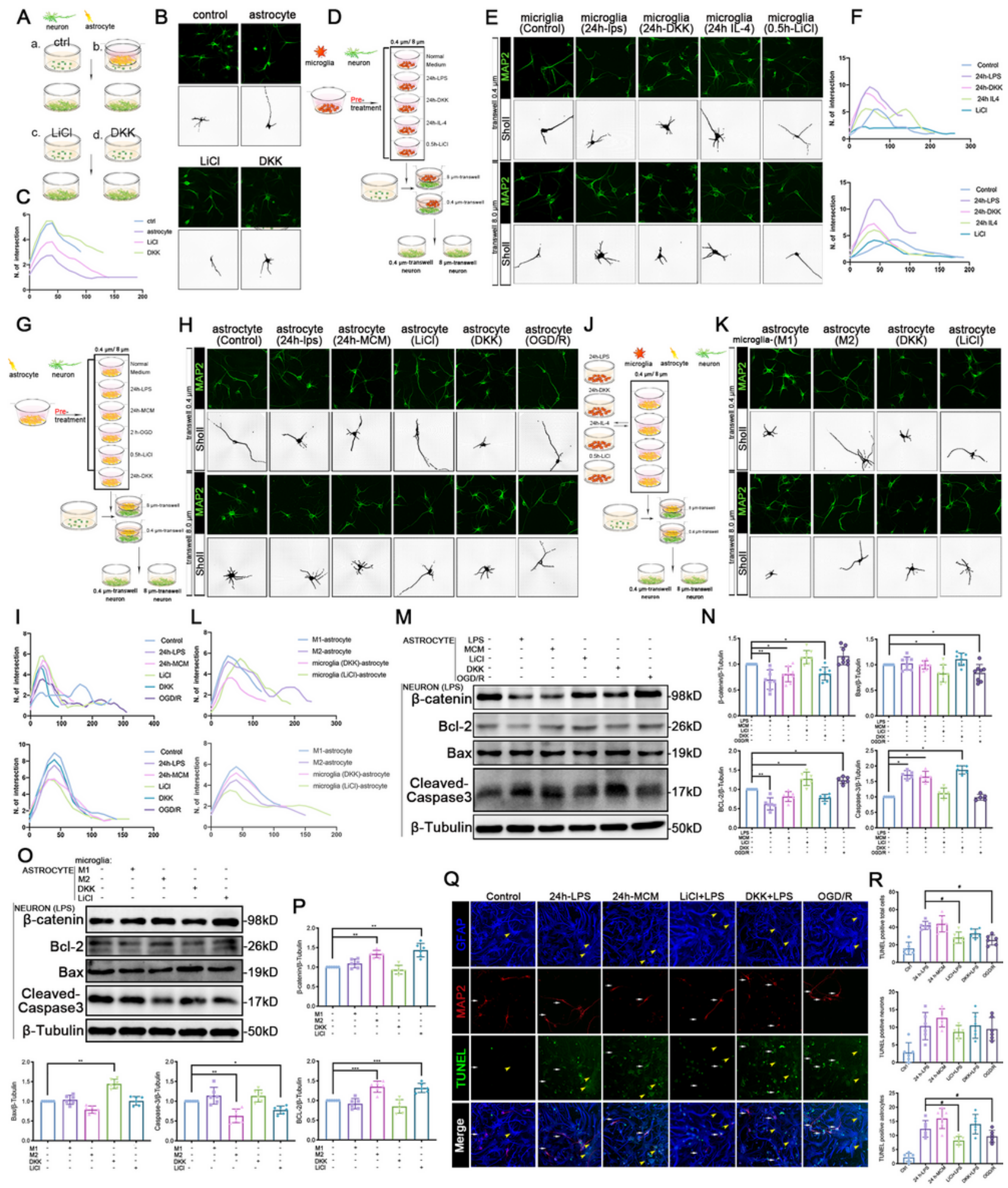


\section{Figure 5}

Activation of WNT signaling in astrocytes promote neuronal maturation. (A) The experimental design for regulation of WNT in primary neuron. (B) Representative images of MAP2 immunostaining of primary cortical neurons. (C) Quantification by Sholl analysis of neurite complexity. (D) The experimental design of migration analysis in co-cultured neurons and microglia. (E) Representative images of MAP2 immunostaining primary cortical neurons. (F) Quantification by Sholl analysis of neurite complexity. (G) The experimental design of migration analysis in co-cultured neurons and astrocytes. $(H)$ Representative images of MAP2 immunostaining of primary cortical neurons. (I) Quantification by Sholl analysis of neurite complexity. $(\mathrm{J})$ The experimental design of migration analysis in co-cultured neurons, astrocytes and microglia. (K) Representative images of MAP2 immunostaining of primary cortical neurons. (L) Quantification by Sholl analysis of neurite complexity. (M) The level of $\beta$-catenin and apoptosis related proteins (Bcl-2, Bax and Caspase 3 ) in primary neurons $8 \mathrm{~h}$ after LPS treatment. $(\mathrm{N}){ }^{*} \mathrm{p}<0.05,{ }^{*} \mathrm{p}<0.01 \mathrm{vs}$ control astrocytes co-culture group ( $n=3$ independent cells preparations). ( 0 ) The level of $\beta$-catenin and apoptosis related protein (Bcl-2, Bax and Caspase 3 ) in neurons $8 \mathrm{~h}$ after conditioned treatment of microglia-astrocyte-neuron axis. $(P){ }^{*}<<0.05, * \star p<0.01$ vs control astrocytes co-culture group $(n=3$ independent cells preparations). (Q) TUNEL assay on co-cultured astrocytes and neurons model after LPS, activated-MCM, LiCl+LPS, DKK+LPS and OGD/R treatment, cells nuclei are strained with DAPI (blue), and apoptotic cells are labeled with TUNEL (green). (R)\#p<0.05, \#\#p<0.01 vs injury (LPS treatment) group ( $n=3$ independent cells preparations). 
A

\section{PHENOTYPE AND POLARIZATION}

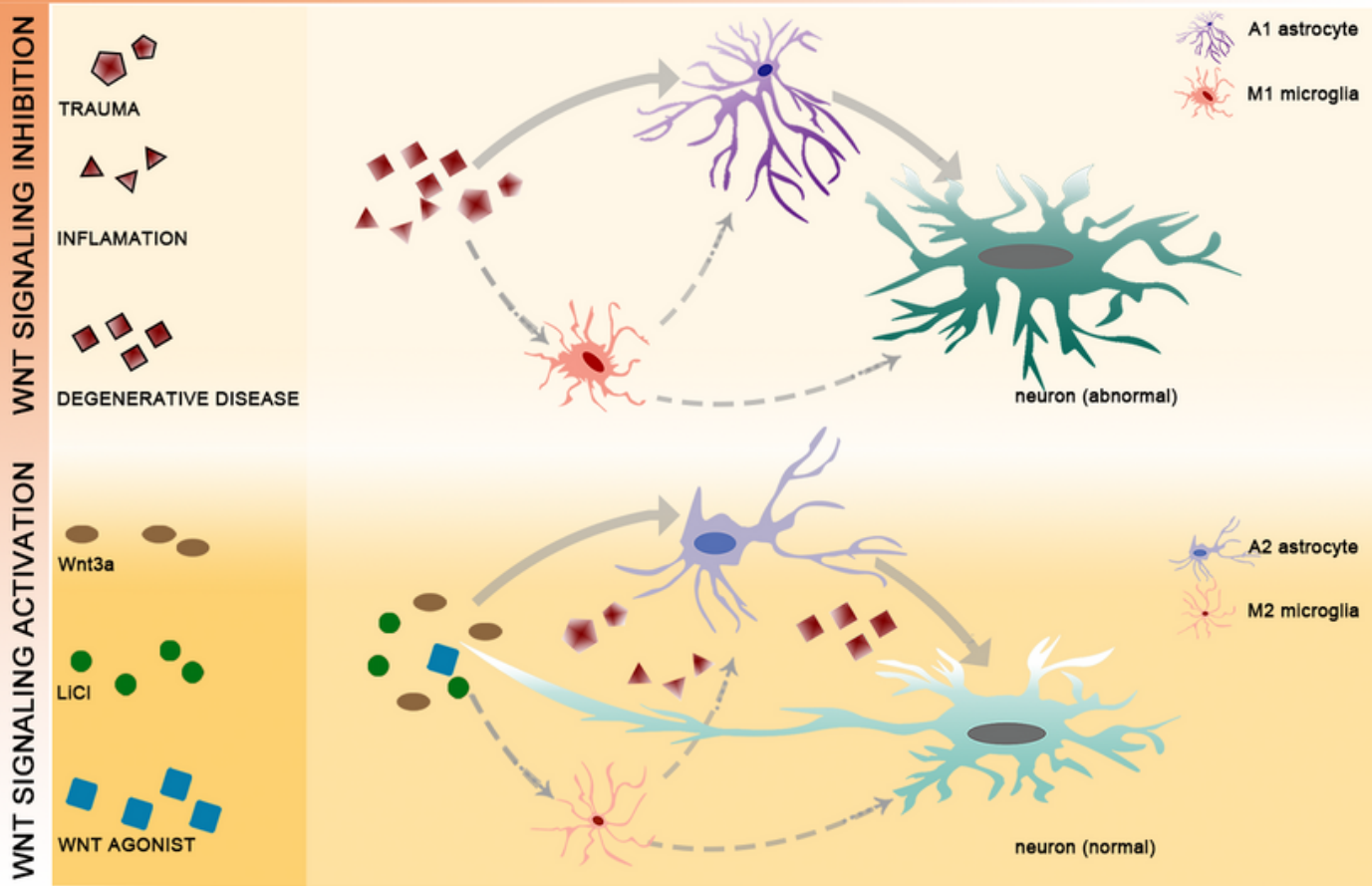

B

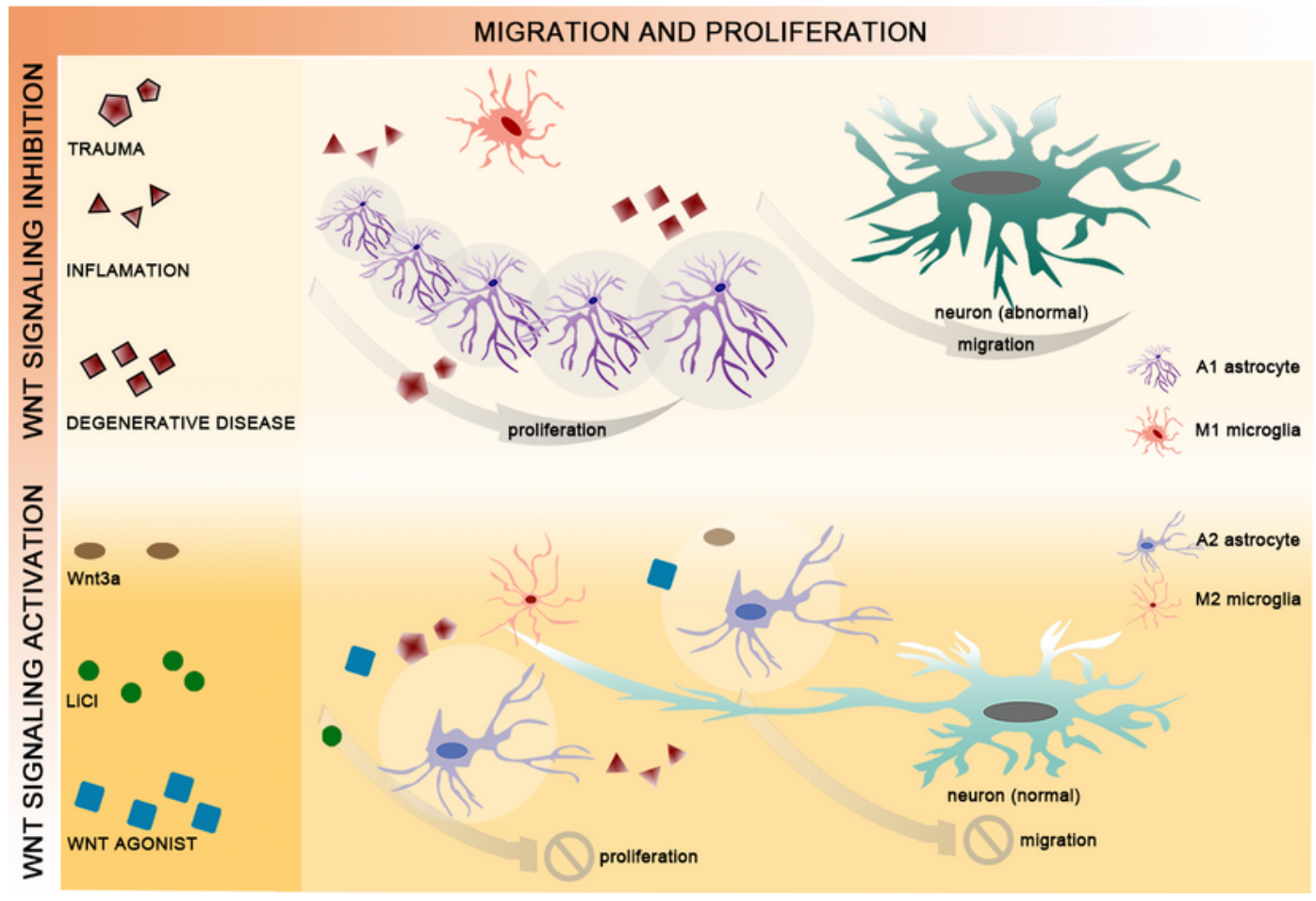

\section{Figure 6}

Neuroprotective mechanism diagram of WNT signaling pathway on CNS trauma and diseases. The neuronal function is impaired after TBI, brain inflammation and degenerative disease. Injured neurons promote astrocytes migration and proliferation which induce the formation of glial scar, and it is an essential protection mechanism. However, the injury could directly stimulate resting astrocyte and generate A1-reactivate astrocyte which is hazard for regulation of neuron, in the meantime, M1-microglia 
could also induce generation of A1-astrocytes to aggravate damage. Activation of WNT/ $\beta$-catenin signaling pathway is beneficial to formation of $A 2$ astrocytes which promote effect of neuroprotective, and M2-microglia could be also induced by WNT pathway which contribute to maintain A2-or resting astrocytes to exert neuroprotective mechanism. Moreover, the activation of WNT could accelerate synaptic elimination and maintain functional integrity of neurons.

\section{Supplementary Files}

This is a list of supplementary files associated with this preprint. Click to download.

- Supplementarymaterial.docx 